\title{
On a new application of almost increasing sequences
}

\section{HS Özarslan and A Keten*}

\section{"Correspondence:}

keten@erciyes.edu.tr

Department of Mathematics, Erciyes

University, Kayseri, 38039, Turkey

\begin{abstract}
In (Bor in Int. J. Math. Math. Sci. 17:479-482, 1994), Bor has proved the main theorem dealing with $\left|\bar{N}, p_{n}\right|_{k}$ summability factors of an infinite series. In the present paper, we have generalized this theorem on the $\varphi-\left|A, p_{n}\right|_{k}$ summability factors under weaker conditions by using an almost increasing sequence instead of a positive non-decreasing sequence.
\end{abstract}

MSC: 40D15; 40F05; 40G99

Keywords: absolute matrix summability; almost increasing sequences; infinite series

\section{Introduction}

Let $\sum a_{n}$ be a given infinite series with the partial sums $\left(s_{n}\right)$. We denote by $t_{n}$ the $n$th $(C, 1)$ mean of the sequence $\left(s_{n}\right)$. The series $\sum a_{n}$ is said to be summable $|C, 1|_{k}, k \geq 1$, if (see [1])

$$
\sum_{n=1}^{\infty} n^{k-1}\left|t_{n}-t_{n-1}\right|^{k}<\infty
$$

Let $\left(p_{n}\right)$ be a sequence of positive numbers such that

$$
P_{n}=\sum_{v=0}^{n} p_{v} \rightarrow \infty \quad \text { as } n \rightarrow \infty\left(P_{-i}=p_{-i}=0, i \geq 1\right)
$$

The sequence-to-sequence transformation

$$
\sigma_{n}=\frac{1}{P_{n}} \sum_{v=0}^{n} p_{v} s_{v}
$$

defines the sequence $\left(\sigma_{n}\right)$ of the $\left(\bar{N}, p_{n}\right)$ mean of the sequence $\left(s_{n}\right)$, generated by the sequence of coefficients $\left(p_{n}\right)$ (see [2]). The series $\sum a_{n}$ is said to be summable $\left|\bar{N}, p_{n}\right|_{k}, k \geq 1$, if (see [3])

$$
\sum_{n=1}^{\infty}\left(\frac{P_{n}}{p_{n}}\right)^{k-1}\left|\sigma_{n}-\sigma_{n-1}\right|^{k}<\infty
$$

Let $A=\left(a_{n v}\right)$ be a normal matrix, i.e., a lower triangular matrix of nonzero diagonal entries. Then $A$ defines the sequence-to-sequence transformation, mapping the sequence

\section{Springer}

(c) 2013 Özarslan and Keten; licensee Springer. This is an Open Access article distributed under the terms of the Creative Commons Attribution License (http://creativecommons.org/licenses/by/2.0), which permits unrestricted use, distribution, and reproduction in any medium, provided the original work is properly cited. 
$s=\left(s_{n}\right)$ to $A s=\left(A_{n}(s)\right)$, where

$$
A_{n}(s)=\sum_{v=0}^{n} a_{n v} s_{v}, \quad n=0,1, \ldots
$$

The series $\sum a_{n}$ is said to be summable $\left|A, p_{n}\right|_{k}, k \geq 1$, if (see [4])

$$
\sum_{n=1}^{\infty}\left(\frac{P_{n}}{p_{n}}\right)^{k-1}\left|\bar{\Delta} A_{n}(s)\right|^{k}<\infty,
$$

where

$$
\bar{\Delta} A_{n}(s)=A_{n}(s)-A_{n-1}(s) .
$$

Let $\left(\varphi_{n}\right)$ be any sequence of positive real numbers. The series $\sum a_{n}$ is said to be summable $\varphi-\left|A, p_{n}\right|_{k}, k \geq 1$, if (see [5])

$$
\sum_{n=1}^{\infty} \varphi_{n}^{k-1}\left|\bar{\Delta} A_{n}(s)\right|^{k}<\infty
$$

If we take $\varphi_{n}=\frac{p_{n}}{p_{n}}$, then $\varphi-\left|A, p_{n}\right|_{k}$ summability reduces to $\left|A, p_{n}\right|_{k}$ summability. Also, if we take $\varphi_{n}=\frac{p_{n}}{p_{n}}$ and $a_{n v}=\frac{p_{v}}{P_{n}}$, then we get $\left|\bar{N}, p_{n}\right|_{k}$ summability. Furthermore, if we take $\varphi_{n}=n, a_{n v}=\frac{p_{v}}{P_{n}}$ and $p_{n}=1$ for all values of $n, \varphi-\left|A, p_{n}\right|_{k}$ reduces to $|C, 1|_{k}$ summability. Finally, if we take $\varphi_{n}=n$ and $a_{n v}=\frac{p_{v}}{P_{n}}$, then we get $\left|R, p_{n}\right|_{k}$ summability (see [6]).

Before stating the main theorem, we must first introduce some further notations.

Given a normal matrix $A=\left(a_{n v}\right)$, we associate two lower semimatrices $\bar{A}=\left(\bar{a}_{n v}\right)$ and $\hat{A}=\left(\hat{a}_{n v}\right)$ as follows:

$$
\bar{a}_{n v}=\sum_{i=v}^{n} a_{n i}, \quad n, v=0,1, \ldots
$$

and

$$
\hat{a}_{00}=\bar{a}_{00}=a_{00}, \quad \hat{a}_{n v}=\bar{a}_{n v}-\bar{a}_{n-1, v}, \quad n=1,2, \ldots
$$

It may be noted that $\bar{A}$ and $\hat{A}$ are the well-known matrices of series-to-sequence and seriesto-series transformations, respectively. Then we have

$$
A_{n}(s)=\sum_{v=0}^{n} a_{n v} s_{v}=\sum_{v=0}^{n} \bar{a}_{n v} a_{v}
$$

and

$$
\bar{\Delta} A_{n}(s)=\sum_{\nu=0}^{n} \hat{a}_{n v} a_{\nu}
$$

\section{Known result}

Many works have been done dealing with $\left|\bar{N}, p_{n}\right|_{k}$ summability factors of infinite series (see [7-22]). Among them, in [21], the following main theorem has been proved. 
Theorem A Let $\left(X_{n}\right)$ be a positive non-decreasing sequence and let there be sequences $\left(\beta_{n}\right)$ and $\left(\lambda_{n}\right)$ such that

$$
\begin{aligned}
& \left|\Delta \lambda_{n}\right| \leq \beta_{n}, \\
& \beta_{n} \rightarrow 0 \quad \text { as } n \rightarrow \infty, \\
& \left|\lambda_{n}\right| X_{n}=O(1) \quad \text { as } n \rightarrow \infty, \\
& \sum_{n=1}^{\infty} n\left|\Delta \beta_{n}\right| X_{n}<\infty
\end{aligned}
$$

are satisfied. Furthermore, if $\left(p_{n}\right)$ is a sequence of positive numbers such that

$$
\begin{aligned}
& P_{n}=O\left(n p_{n}\right) \quad \text { as } n \rightarrow \infty, \\
& \sum_{n=1}^{m} \frac{p_{n}}{P_{n}}\left|s_{n}\right|^{k}=O\left(X_{m}\right) \quad \text { as } m \rightarrow \infty,
\end{aligned}
$$

then the series $\sum a_{n} \lambda_{n}$ is summable $\left|\bar{N}, p_{n}\right|_{k}, k \geq 1$.

\section{The main result}

The aim of this paper is to generalize Theorem $\mathrm{A}$ for $\varphi-\left|A, p_{n}\right|_{k}$ summability under weaker conditions. For this, we need the concept of an almost increasing sequence. A positive sequence $\left(c_{n}\right)$ is said to be almost increasing if there exists a positive increasing sequence $\left(b_{n}\right)$ and two positive constants A and B such that $A b_{n} \leq c_{n} \leq B b_{n}$ (see [23]). Obviously, every increasing sequence is an almost increasing sequence but the converse need not be true as can be seen from the example $b_{n}=n e^{(-1)^{n}}$. Also, one can find some results dealing with absolute almost convergent sequences (see [24]). So, we are weakening the hypotheses of Theorem A replacing the increasing sequence by an almost increasing sequence. Now, we shall prove the following theorem.

Theorem Let $A=\left(a_{n v}\right)$ be a positive normal matrix such that

$$
\begin{aligned}
& \bar{a}_{n 0}=1, \quad n=0,1, \ldots, \\
& a_{n-1, v} \geq a_{n v} \quad \text { for } n \geq v+1, \\
& a_{n n}=O\left(\frac{p_{n}}{P_{n}}\right), \\
& \left|\hat{a}_{n, v+1}\right|=O\left(v\left|\Delta_{v} \hat{a}_{n v}\right|\right) .
\end{aligned}
$$

Let $\left(X_{n}\right)$ be an almost increasing sequence and $\left(\frac{\varphi_{n} p_{n}}{P_{n}}\right)$ be a non-increasing sequence. If conditions (12)-(16) and

$$
\sum_{n=1}^{m} \varphi_{n}^{k-1}\left(\frac{p_{n}}{P_{n}}\right)^{k}\left|s_{n}\right|^{k}=O\left(X_{m}\right) \quad \text { as } m \rightarrow \infty,
$$

are satisfied, then the series $\sum a_{n} \lambda_{n}$ is summable $\varphi-\left|A, p_{n}\right|_{k}, k \geq 1$. 
Remark It should be noted that if we take $\left(X_{n}\right)$ as a positive non-decreasing sequence, $\varphi_{n}=\frac{P_{n}}{p_{n}}$ and $a_{n v}=\frac{p_{v}}{P_{n}}$, then we get Theorem A. In this case, conditions (21) and (22) reduce to conditions (16) and (17), respectively. Also, the condition ' $\left(\frac{\varphi_{n} p_{n}}{P_{n}}\right)$ is a non-increasing sequence' and the conditions (18)-(20) are automatically satisfied.

Lemma [22] Under the conditions on $\left(X_{n}\right),\left(\beta_{n}\right)$ and $\left(\lambda_{n}\right)$ as taken in the statement of the theorem, we have the following:

$$
\begin{aligned}
& n \beta_{n} X_{n}=O(1) \quad \text { as } n \rightarrow \infty, \\
& \sum_{n=1}^{\infty} \beta_{n} X_{n}<\infty .
\end{aligned}
$$

Proof of the Theorem Let $\left(T_{n}\right)$ denote $A$-transform of the series $\sum a_{n} \lambda_{n}$. Then we have, by (10) and (11),

$$
\bar{\Delta} T_{n}=\sum_{v=1}^{n} \hat{a}_{n v} \lambda_{\nu} a_{v} .
$$

Applying Abel's transformation to this sum, we get that

$$
\begin{aligned}
\bar{\Delta} T_{n} & =\sum_{v=1}^{n-1} \Delta_{v}\left(\hat{a}_{n v} \lambda_{v}\right) s_{v}+\hat{a}_{n n} \lambda_{n} s_{n} \\
& =\sum_{v=1}^{n-1}\left(\hat{a}_{n v} \lambda_{v}-\hat{a}_{n, v+1} \lambda_{v+1}\right) s_{v}+\hat{a}_{n n} \lambda_{n} s_{n} \\
& =\sum_{v=1}^{n-1} \Delta_{v}\left(\hat{a}_{n v}\right) \lambda_{v} s_{v}+\sum_{v=1}^{n-1} \hat{a}_{n, v+1} s_{v} \Delta \lambda_{v}+a_{n n} \lambda_{n} s_{n} \\
& =T_{n}(1)+T_{n}(2)+T_{n}(3) .
\end{aligned}
$$

To complete the proof of the theorem, by Minkowski's inequality, it is sufficient to show that

$$
\sum_{n=1}^{\infty} \varphi_{n}^{k-1}\left|T_{n}(r)\right|^{k}<\infty \quad \text { for } r=1,2,3
$$

Now, when $k>1$, applying Hölder's inequality with indices $k$ and $k$, where $1 / k+1 / k=1$, we have that

$$
\begin{aligned}
\sum_{n=2}^{m+1} \varphi_{n}^{k-1}\left|T_{n}(1)\right|^{k} & =O(1) \sum_{n=2}^{m+1} \varphi_{n}^{k-1}\left(\sum_{v=1}^{n-1}\left|\Delta_{v}\left(\hat{a}_{n v}\right)\right|\left|\lambda_{v}\right|\left|s_{v}\right|\right)^{k} \\
& =O(1) \sum_{n=2}^{m+1} \varphi_{n}^{k-1}\left(\sum_{v=1}^{n-1}\left|\Delta_{v}\left(\hat{a}_{n v}\right)\right|\left|\lambda_{v}\right|^{k}\left|s_{v}\right|^{k}\right) \times\left(\sum_{v=1}^{n-1}\left|\Delta_{v}\left(\hat{a}_{n v}\right)\right|\right)^{k-1} \\
& =O(1) \sum_{n=2}^{m+1}\left(\frac{\varphi_{n} p_{n}}{P_{n}}\right)^{k-1}\left(\sum_{v=1}^{n-1}\left|\Delta_{v}\left(\hat{a}_{n v}\right)\right|\left|\lambda_{v}\right|^{k}\left|s_{v}\right|^{k}\right)
\end{aligned}
$$




$$
\begin{aligned}
& =O(1) \sum_{v=1}^{m}\left|\lambda_{v}\right|^{k}\left|s_{v}\right|^{k} \sum_{n=v+1}^{m+1}\left(\frac{\varphi_{n} p_{n}}{P_{n}}\right)^{k-1}\left|\Delta_{v}\left(\hat{a}_{n v}\right)\right| \\
& =O(1) \sum_{v=1}^{m}\left|\lambda_{v}\right|^{k}\left|s_{v}\right|^{k}\left(\frac{\varphi_{v} p_{v}}{P_{v}}\right)^{k-1} \sum_{n=v+1}^{m+1}\left|\Delta_{v}\left(\hat{a}_{n v}\right)\right| \\
& =O(1) \sum_{v=1}^{m}\left|\lambda_{v}\right|^{k-1}\left|\lambda_{v}\right|\left|s_{v}\right|^{k}\left(\frac{\varphi_{v} p_{v}}{P_{v}}\right)^{k-1}\left(\frac{p_{v}}{P_{v}}\right) \\
& =O(1) \sum_{v=1}^{m}\left|\lambda_{v}\right| \varphi_{v}^{k-1}\left(\frac{p_{v}}{P_{v}}\right)^{k}\left|s_{v}\right|^{k} \\
& =O(1) \sum_{v=1}^{m-1} \Delta\left|\lambda_{v}\right| \sum_{r=1}^{v} \varphi_{r}^{k-1}\left(\frac{p_{r}}{P_{r}}\right)^{k}\left|s_{r}\right|^{k}+O(1)\left|\lambda_{m}\right| \sum_{v=1}^{m} \varphi_{v}^{k-1}\left(\frac{p_{v}}{P_{v}}\right)^{k}\left|s_{v}\right|^{k} \\
& =O(1) \sum_{v=1}^{m-1}\left|\Delta \lambda_{v}\right| X_{v}+O(1)\left|\lambda_{m}\right| X_{m} \\
& =O(1) \sum_{v=1}^{m-1} \beta_{v} X_{v}+O(1)\left|\lambda_{m}\right| X_{m} \\
& =O(1) \quad \text { as } m \rightarrow \infty
\end{aligned}
$$

by virtue of the hypotheses of the theorem and the lemma. Again, applying Hölder's inequality and using the fact that $v \beta_{v}=O\left(\frac{1}{X_{\nu}}\right)=O(1)$ by (23), we get that

$$
\begin{aligned}
\sum_{n=2}^{m+1} \varphi_{n}^{k-1}\left|T_{n}(2)\right|^{k} & =O(1) \sum_{n=2}^{m+1} \varphi_{n}^{k-1}\left(\sum_{v=1}^{n-1}\left|\hat{a}_{n, v+1}\right|\left|\Delta \lambda_{v}\right|\left|s_{v}\right|\right)^{k} \\
& =O(1) \sum_{n=2}^{m+1} \varphi_{n}^{k-1}\left(\sum_{v=1}^{n-1}\left|\hat{a}_{n, v+1}\right| \beta_{v}\left|s_{v}\right|^{k}\right) \times\left(\sum_{v=1}^{n-1}\left|\hat{a}_{n, v+1}\right| \beta_{v}\right)^{k-1} \\
& =O(1) \sum_{n=2}^{m+1} \varphi_{n}^{k-1}\left(\sum_{v=1}^{n-1}\left|\hat{a}_{n, v+1}\right| \beta_{v}\left|s_{v}\right|^{k}\right) \times\left(\sum_{v=1}^{n-1} v\left|\Delta_{v}\left(\hat{a}_{n v}\right)\right| \beta_{v}\right)^{k-1} \\
& =O(1) \sum_{n=2}^{m+1}\left(\frac{\varphi_{n} p_{n}}{P_{n}}\right)^{k-1}\left(\sum_{v=1}^{n-1} v\left|\Delta_{v}\left(\hat{a}_{n v}\right)\right| \beta_{v}\left|s_{v}\right|^{k}\right) \\
& =O(1) \sum_{v=1}^{m} v \beta_{v}\left|s_{v}\right|^{k} \sum_{n=v+1}^{m+1}\left(\frac{\varphi_{n} p_{n}}{P_{n}}\right)^{k-1}\left|\Delta_{v}\left(\hat{a}_{n v}\right)\right| \\
& =O(1) \sum_{v=1}^{m} v \beta_{v}\left|s_{v}\right|^{k}\left(\frac{\varphi_{v} p_{v}}{P_{v}}\right)^{k-1} \sum_{n=v+1}^{m+1}\left|\Delta_{v}\left(\hat{a}_{n v}\right)\right| \\
& =O(1) \sum_{v=1}^{m} v \beta_{v}\left|s_{v}\right|^{k}\left(\frac{\varphi_{v} p_{v}}{P_{v}}\right)^{k-1}\left(\frac{p_{v}}{P_{v}}\right) \\
& =O(1) \sum_{v=1}^{m-1} \Delta\left(v \beta_{v}\right) \sum_{r=1}^{v} \varphi_{r}^{k-1}\left(\frac{p_{r}}{P_{r}}\right)^{k}\left|s_{r}\right|^{k}+O(1) m \beta_{m} \sum_{v=1}^{m} \varphi_{v}^{k-1}\left(\frac{p_{v}}{P_{v}}\right)^{k}\left|s_{v}\right|^{k} \\
& O(1) \sum_{v=1}^{m-1}\left|\Delta\left(v \beta_{v}\right)\right| X_{v}+O(1) m \beta_{m} X_{m}
\end{aligned}
$$




$$
\begin{aligned}
& =O(1) \sum_{v=1}^{m-1} v\left|\Delta \beta_{v}\right| X_{v}+O(1) \sum_{v=1}^{m-1} \beta_{v+1} X_{v+1}+O(1) m \beta_{m} X_{m} \\
& =O(1) \quad \text { as } m \rightarrow \infty
\end{aligned}
$$

by virtue of the hypotheses of the theorem and the lemma. Finally, as in $T_{n}(1)$, we have that

$$
\begin{aligned}
\sum_{n=1}^{m} \varphi_{n}^{k-1}\left|T_{n}(3)\right|^{k} & =O(1) \sum_{n=1}^{m} \varphi_{n}^{k-1}\left|a_{n n} \lambda_{n} s_{n}\right|^{k} \\
& =O(1) \sum_{n=1}^{m}\left|\lambda_{n}\right| \varphi_{n}^{k-1}\left(\frac{p_{n}}{P_{n}}\right)^{k}\left|s_{n}\right|^{k} \\
& =O(1) \quad \text { as } m \rightarrow \infty .
\end{aligned}
$$

This completes the proof of the theorem. If we take $\varphi_{n}=\frac{P_{n}}{p_{n}}$, then we get a result concerning the $\left|A, p_{n}\right|_{k}$ summability factors. If we take $a_{n v}=\frac{p_{v}}{P_{n}}$, then we have another result dealing with $\left|\bar{N}, p_{n}, \varphi_{n}\right|_{k}$ summability. If we take $a_{n v}=\frac{p_{v}}{P_{n}}$ and $p_{n}=1$ for all values of $\mathrm{n}$, then we get a result dealing with $\left|C, 1, \varphi_{n}\right|_{k}$ summability. If we take $\varphi_{n}=n, a_{n v}=\frac{p_{v}}{P_{n}}$ and $p_{n}=1$ for all values of $\mathrm{n}$, then we get a result for $|C, 1|_{k}$ summability.

\section{Competing interests}

The authors declare that they have no competing interests.

\section{Authors' contributions}

All authors contributed equally to the manuscript and read and approved the final manuscript.

Received: 3 September 2012 Accepted: 6 November 2012 Published: 9 January 2013

\section{References}

1. Flett, TM: On an extension of absolute summability and some theorems of Littlewood and Paley. Proc. Lond. Math. Soc. 7, 113-141 (1957)

2. Hardy, GH: Divergent Series. Oxford University Press, Oxford (1949)

3. Bor, H: On two summability methods. Math. Proc. Camb. Philos. Soc. 97, 147-149 (1985)

4. Sulaiman, WT: Inclusion theorems for absolute matrix summability methods of an infinite series (IV). Indian J. Pure Appl. Math. 34(11), 1547-1557 (2003)

5. Özarslan, HS, Keten, A: A new application of almost increasing sequences. An. ştiinţ. Univ. "Al.I. Cuza" laşi, Mat. (2012, in press)

6. Bor, $\mathrm{H}$ : On the relative strength of two absolute summability methods. Proc. Am. Math. Soc. 113, 1009-1012 (1991)

7. Bor, H: On $\left|\bar{N}, p_{n}\right|_{k}$ summability factors. Proc. Am. Math. Soc. 94, 419-422 (1985)

8. Bor, H: A note on $\left|\bar{N}, p_{n}\right|_{k}$ summability factors of infinite series. Indian J. Pure Appl. Math. 18, 330-336 (1987)

9. Bor, H: On absolute summability factors. Analysis 7, 185-193 (1987)

10. Bor, H: Absolute summability factors for infinite series. Indian J. Pure Appl. Math. 19, 664-671 (1988)

11. Bor, $\mathrm{H}$, Kuttner, B: On the necessary conditions for absolute weighted arithmetic mean summability factors. Acta Math. Hung. 54, 57-61 (1989)

12. Bor, $\mathrm{H}$ : A note on $\left|\bar{N}, p_{n}\right|_{k}$ summability factors. Bull. Calcutta Math. Soc. 82, 357-362 (1990)

13. Bor, H: Absolute summability factors for infinite series. Math. Jpn. 36, 215-219 (1991)

14. Bor, $H$ : Factors for $\left|\bar{N}, p_{n}\right|_{k}$ summability of infinite series. Ann. Acad. Sci. Fenn., Ser. A 1 Math. 16, 151-154 (1991)

15. Bor, $\mathrm{H}$ : On absolute summability factors for $\left|\bar{N}, p_{n}\right|_{k}$ summability. Comment. Math. Univ. Carol. 32(3), 435-439 (1991)

16. Bor, $\mathrm{H}$ : On the $\left|\bar{N}, p_{n}\right|_{k}$ summability factors for infinite series. Proc. Indian Acad. Sci. Math. Sci. 101, 143-146 (1991)

17. Bor, $\mathrm{H}$ : A note on $\left|\bar{N}, p_{n}\right|_{k}$ summability factors. Rend. Mat. Appl. (7) 12, 937-942 (1992)

18. Bor, H: On absolute summability factors. Proc. Am. Math. Soc. 118, 71-75 (1993)

19. Bor, H: On the absolute Riesz summability factors. Rocky Mt. J. Math. 24, 1263-1271 (1994)

20. Bor, H: On $\left|\bar{N}, p_{n}\right|_{k}$ summability factors. Kuwait J. Sci. Eng. 23, 1-5 (1996)

21. Bor, H: A note on absolute summability factors. Int. J. Math. Math. Sci. 17, 479-482 (1994)

22. Mazhar, SM: A note on absolute summability factors. Bull. Inst. Math. Acad. Sin. 25(3), 233-242 (1997)

23. Bari, NK, Stečkin, SB: Best approximation and differential properties of two conjugate functions. Tr. Mosk. Mat. Obŝ. 5 , 483-522 (1956) (in Russian)

24. Çakalli, H, Çanak, G: ( $\left.p_{n}, s\right)$-absolute almost convergent sequences. Indian J. Pure Appl. Math. 28(4), 525-532 (1997) 
doi:10.1186/1029-242X-2013-13

Cite this article as: Özarslan and Keten: On a new application of almost increasing sequences. Journal of Inequalities and Applications 2013 2013:13.

Submit your manuscript to a SpringerOpen ${ }^{\circ}$ journal and benefit from:

- Convenient online submission

- Rigorous peer review

- Immediate publication on acceptance

Open access: articles freely available online

- High visibility within the field

- Retaining the copyright to your article

Submit your next manuscript at $\boldsymbol{s p r i n g e r o p e n . c o m ~}$ 